\title{
La sécurité alimentaire : la construction d'un bien public global ?
}

\author{
François LERIN ${ }^{1}$ \\ Sélim LOUAFI ${ }^{2}$ \\ ${ }^{1}$ Institut Agronomique Méditerranéen \\ de Montpellier (CIHEAM-IAMM), \\ 3191 route de Mende 34093 \\ Montpellier cedex 5, \\ France \\ <lerin@iamm.fr> \\ 2 Cirad, \\ UMR AGAP, \\ F-34398 Montpellier, France
}

Article reçu le 25 juillet 2012

Accepté le 3 août 2012

\begin{abstract}
Food security: the building of a common public share?
Soaring of food prices in 2007/08 has put again food security high in the global agenda. Several elements of explanation have been put forward to explain this crisis, including traditional ones such as imbalance supply/demand and stocks, energy effect, dollar effect; and new factors such as biofuels, financial speculation and low interest rates, export bans. Various arguments linked to this food crisis have also questioned the global nature of food security issue : global insecurity (with the urban food riots), humanitarian (with the worsening situation of the bottom billion), cross-sectoral (with the relationships between agriculture and other related issues such as environment, health, trade rules and market impacts, etc.). Several initiatives have insisted on the need to address more seriously the problem at the global level. Indeed, lack of global coordination on food security has been recognized as a major problem. This lack of coordination could be understood both at the substantive level (i.e. between the different issue-areas which are part of the food security problem) and at the institutional level (i.e. between the different international organizations in charge of food security) leading to the fragmentation of global food security governance. This paper respectively analyses these two dimensions of fragmentation in order to assess the extent to which current global initiatives better address coordination needs for global food security.
\end{abstract}

Key words: global food security, fragmentation, global governance, discourse, international organizations

choc pétrolier (de Janvry, Sadoulet, 2008 ; Gérard et al., 2008 ; Mitchell, 2008 ; Testard-Vailland, 2008 ; Timmer, 2010).

Comme on le sait, cette hausse spectaculaire a été suivie $d^{\prime}$ une baisse tout aussi spectaculaire des prix effondrés par la crise économique financière mondiale. De nombreux facteurs - certains classiques, d'autres plus spécifiques - ont été mis à jour par les analystes dans une abondante littérature descriptive (Abbot et al., 2008 ; von Braun, 2007, 2008 ; de Janvry et Sadoulet, 2008 ; EC, 2008 ; IATP, 2008 ; Lerin et al., 2009 ; Mitchell, 2008 ; Piesse et al., 2009 ; Robles et al., 2009 ; Trostle, 2008 ; Voituriez, 2009). Les raisons invoquées pour expliquer cette hausse (spéculation, biocarbu- rants, demande des pays émergents, effet dollar, etc.) sont encore sujets à contestations et travaux. En revanche, il y a eu une certaine convergence pour annoncer: d'une part, que les prix des matières premières agricoles seraient au cours des prochaines années orientés par une hausse structurelle et que, d'autre part, on devrait s'attendre à plus de "volatilité ", c'est-à-dire de variations brusques largement imprévisibles (FAO et OECD, 2011 ; Brunel et al., 2009 ; Evans, 2009). C'est ce qui s'est en effet passé et I'on assiste à une variabilité de plus en plus importante des prix des denrées agricoles - cette fois non plus conjointement mais marché par marché en fonction des conjonctures d'offre et de demande - comme on peut le constater au cours de l'été 2012 avec la

Pour citer cet article : Lerin F, Louafi S. La sécurité alimentaire : la construction d'un bien public global ? OCL $2012 ; 19(5)$ : $276-282$. doi : 10.1684/ocl.2012.0473 
pression à la hausse sur les grains de base (mais, blé, soja) provoquée par une sécheresse historique aux États-Unis qui a conduit le secrétaire d'État à l'Agriculture, Tom Vilslack, à déclarer l'état de catastrophe naturelle dans plus de 1000 comtés du pays.

Mais I'instabilité des prix n'est dans cette discussion sur ce que I'on appelle de plus en plus systématiquement la " sécurité alimentaire mondiale " qu'un des aspects d'une question multiforme touchant à la fois à la sécurité des aliments, aux marchés et au commerce mondial, à la lutte contre la pauvreté, à la vulnérabilité de certains économie et de certaines couches sociales, aux situations de crises climatiques ou politico-militaires, etc. L'ambiguïté de certaines prises de position et les incertitudes liées au déploiement d'une coordination internationale sur le thème tient pour partie dans ce fait qu'il recouvre des problèmes de natures différentes, affectant de manière spécifique à la fois des régions et des populations et des économies. Il n'en reste pas moins que cette ambiguiité est admise et largement reconnue et que la crise de 2008/2009 a été un moment important $d^{\prime} u n$ mouvement d'idées et d'organisation pour constituer, progressivement et de façon contrariée et complexe, la question alimentaire comme une question de statut global, un objet de coordination internationale (Bricas et Daviron, 2008 ; Margulis, 2009, 2011 ; Lerin et al., 2009 ; Oxfam, 2009 ; Shaw, 2009).

Mais jusqu'où et comment cette question de la sécurité alimentaire est-elle une question internationale ? La réponse est loin d'être évidente. L'approvisionnement alimentaire des populations est un enjeu fondateur des régimes politiques modernes. L'inclusion des pauvres et des "crève-la-faim ", la "police des grains ", I'approvisionnement des villes, la lutte contre les disettes, la nourriture des pauvres et des ouvriers sont des questions fondamentales de la souveraineté et de la citoyenneté. L'idée donc que la sécurité alimentaire est une question internationale ne va pas de soi, étant donné que le principe de souveraineté est au cœur du système des relations internationales et qu'il n'existe, encore aujourd'hui, qu'un vague embryon de citoyenneté internationale. Cette question de la sécurité alimentaire mondiale doit donc être comprise comme une construction rela- tivement récente et toujours " en train de se faire ". Nous allons dans ce court article proposer l'hypothèse que la "sécurité alimentaire " est composée de plusieurs strates - ce que nous avons appelé des socles institutionnels et discursifs - et, d'autre part, faire un état des lieux des coordinations les plus intégratrices qui ont eu lieu au cours de ces années récentes - ce que nous désignons sous le terme de méta-initiatives pour une sécurité alimentaire globale.

\section{La sécurité alimentaire internationale, un complexe d'institutions et de discours}

Ce que l'on regroupe aujourd'hui sous le terme de sécurité alimentaire mondiale est donc un ensemble de questions qui ont émergées dans des crises et qui ont donné lieu, pour y répondre, à la création d'institutions organisées par des mandats, des moyens, des idées et des discussions séparées. Nous avons distingué cinq de ces socles.

Le premier de ces socles qui peut être identifié est celui qui a trait à l'agriculture. Depuis la mondialisation des échanges de produits pondéreux agricoles, la question de la disponibilité des produits a été l'objet de multiples préoccupations, privées ou publiques. Partagé entre les deux hémisphères et entre les cinq continents la production agricole mondiale, et notamment la production agricole exportable de grains, a très tôt été désignée comme une question nécessitant une coordination de l'information : pour connaître les situations de production, évaluer les récoltes et les stocks, estimer les soldes exportables, les besoins et l'offre (Shaw, 2007). Un Bureau de l'agriculture a été crée, en 1905, dans le mouvement de la construction infructueuse de la Société des Nations et la FAO (organisation mondiale de I'agriculture et de l'alimentation des Nations-Unies) a été instituée dès 1943 (McCalla, 2007). La coordination internationale des recherches agronomiques, d'abord autour de fondations privées américaines puis dans des centres de recherche spécialisés par produits (et regroupés dans le CGIAR), la création du Conseil Mondial de I'Alimentation en 1974 ou le Fida en 1978, etc. sont autant d'organisations créées dans cette perspective et qui tentent de faire coïncider la production et la demande mondiale, principalement en œuvrant à l'augmentation de la production (Lele, 2009). Compte tenu de la nécessité d'un approvisionnement sur le marché mondial (ponctuel ou structurel) de nombreuses économies alimentaires, il y a bien, en quelque sorte, une question de l'agriculture mondiale qui peut, doit ou pourrait faire l'objet d'une coordination (information, recherche, politiques) (Bertini et Glickman, 2011 ; Griffon, 2010 ; IFPRI, 2002 ; Jacquet et al., 2011 ; Mclntyre et al., 2009 ; Pretty et al., 2010 ; Shapouri et al., 2010).

Le deuxième socle qui peut être identifié est celui de ce que l'on peut appeler le socle des droits. II existe aujourd'hui un " droit à l'alimentation » qui fait partie des droits économiques et sociaux relié à la Déclaration universelle des droits de I'homme adoptée comme charte constitutive des Nations-Unies en 1948. Ce droit, on le sait et on le déplore parfois, ne dispose pas de juridiction par lequel il serait opposable et donc mis en œuvre. $C^{\prime}$ est un engagement pris par les Nations, au nom des peuples et des individus (Hafner-Burton, 2009 ; RisseKappen, 1999 ; Sen, 2010). C'est I'extension de la Déclaration universelle par le Pacte international relatif aux droits économiques, sociaux et culturels, adopté le 16 décembre 1966 qui définit ce droit à l'alimentation, comme un droit dit " de seconde génération ". En 2000, la Commission des droits de l'homme établissait le mandat d'un Rapporteur spécial sur le droit à l'alimentation au sein des Nations Unies (De Schutter, 2010).

Le troisième socle identifiable est celui qui s'est institué autour des crises humanitaires, que celles-ci soient provoquées par des accidents climatiques ou physiques majeurs ou qu'elles soient I'effet de situations de conflits armés et de guerres internationales ou civiles. Implicitement basées sur le droit des individus (et le " devoir d'ingérence "), des interventions internationales ont été alors conçues pour répondre à ces situations de personnes ou de communautés n'ayant plus d'autorités ou d'État pour les protéger ou pour leur assurer une subsistance minimale (Maxwell et al., 2010 ; Hoddinott et al., 2008). Ces interventions humanitaires $\mathrm{n}^{\prime}$ ont pas seulement pour vocation ou principe de résoudre ou d'alléger des situations d'extrême malnutrition et de famine de populations, réfugiées ou non, elles sont 
aussi, du point de vue des puissances intervenantes, une façon de minimiser les risques politiques des régions affectées. La faim est un puissant facteur de trouble et de révolte. Les interventions sont donc destinées à limiter les déstabilisations et les " contagions " possibles. Ces interventions alimentaires ne se sont d'ailleurs pas limitées aux seules situations $d^{\prime}$ urgence et un certain nombre d'opérations d'aide alimentaire ont été menées dans le cadre du Programme Alimentaire Mondial (PAM - qui s'occupe aussi des situations d'urgence) crée en 1961, pour anticiper ces situations de trouble et apporter des biens de base aux populations les plus pauvres (les Américains l'ont fait au sortir de la guerre pour l'Europe et on poursuivit ces actions sous le titre d'abord de l'Agricultural Trade Development Assistance Act (1954), renommé par le président Kennedy "Food for Peace " et aujourd'hui toujours inclus dans la Farm Bill américaine). L'ensemble des puissances de I'OCDE font de même soit via le PAM soit selon des modalités propres aux dispositifs de coopération et d'aides bilatérales. On peut donc, concernant ces institutions et argumentaires, parler de socle " humanitaire-sécuritaire ".

Le quatrième socle identifiable est celui qui a trait au commerce et aux marchés. En effet, il ne suffit pas que les denrées soient disponibles dans le bilan offre/ demande mondial, encore faut-il que les principes d'organisation du marché assurent la circulation des marchandises et que les marchés, d'un point de vue institutionnel et pratique, soient en mesure de répondre à cette demande. Aujourd'hui la discussion sur la souveraineté alimentaire, comme celle sur la volatilité des prix et la régulation des marchés, oppose des positions très contrastées: les uns voyant dans le marché mondial et sa libéralisation un facteur de déstabilisation des économies agricoles locales (et donc de l'offre) et un mécanisme ne conduisant qu'à la suprématie des grandes agricultures intensives; les autres au contraire soulignant que la circulation des biens alimentaires est une condition sine qua non de la meilleure spécialisation des agricultures mondiales, de l'approvisionnement des populations et donc de la sécurité alimentaire globale (Boussard et al., 2005 ; Davis, 2009 ; Hrabanski, 2011 ; IATP, 2008; Margulis, 2009 ; World Bank, 2009).
Enfin, le dernier socle identifié est celui du développement. Depuis la fin de la deuxième guerre mondiale et plus largement encore depuis les décolonisations, les coopérations internationales (la Banque Mondiale en tout premier lieu) et les coopérations bilatérales ou régionales ont défini des pratiques d'aide au développement, dont une partie non négligeable a d'abord été destinée aux économies agricoles, à l'éradication des situations de famine et ce que l'on appelait à l'époque "la lutte contre la faim ". Même si cette orientation a marqué le pas à partir des années 1990 (elles se sont en fait converties au vocable de la "lutte contre la pauvreté "), ces organisations restent des acteurs décisifs de production de discours, de dispositifs de développement et de moyens qui ont pour ambition affichée aujourd'hui, en luttant contre la pauvreté et pour l'inclusion du " milliard d'en bas " d'être un élément décisif de cette coordination collective pour la sécurité alimentaire globale (Banque mondiale, 2008 ; Drèze et al., 1989; Lofgren et al., 2003 ; Summer, 2010). Les questions nutritionnelles et environnementales y prennent de plus en plus de place.

Ces socles institutionnels et discursifs ne constituent pas un ensemble spontanément coordonné (Alter et Meunier, 2009 ; Arts et Buizer, 2009; Hook, 2001 ; Keohane et Victor, 2011; Phillips et al., 2004 ; Raustiala et Victor, 2004 ; Schmidt, 2008). Même s'il existe des "passages " entre eux et si, dans les situations concrètes, des acteurs et des organisations peuvent, dans un souci d'efficacité de leurs démonstrations, combiner des arguments, ces socles institutionnels et discursifs sont discontinus. Constater cette fragmentation permet de comprendre un peu ce qui est généralement avancé par la littérature comme les raisons de l'inaboutissement perpétuel de l'objectif de sécurité alimentaire à l'échelle globale (Evans, 2009 ; Margulis, 2011 ; Maxwell, 1996 ; Oxfam, 2009) soit: le manque de consensus sur la nature internationale du problème, sur les contours de la question, sur les coordinations nécessaires. Il y a donc de fait à la fois chevauchement et séparation des organisations, des mandats et des intérêts. L'effet de la crise de 2008/2009 et du débat sur la globalisation et ses contempteurs a été de porter cette fragmentation sur le devant de la scène, de provoquer une série d'initiati- ves tentant de trouver des mécanismes susceptibles de contrecarrer les effets négatifs de la fragmentation des institutions traitant de cet ensemble de problèmes.

\section{Les méta-initiatives de coordination}

Le paysage institutionnel international est marqué par un grand nombre d'institutions en charge d'un ou plusieurs aspect(s) de la question de la sécurité alimentaire. Comme nous l'avons vu dans la section précédente, ces institutions sont nées par empilement au fur et à mesure que de nouveaux problèmes se présentaient et même parfois par positionnement explicite contre l'action d'institutions existantes (Lele, 2009; Shaw, 2009).

Ces institutions internationales couvrent un large spectre d'arrangements institutionnels allant de traités ou conventions sur certains aspects particuliers de la sécurité alimentaire, d'agences intergouvernementales onusiennes, d'organisations internationales hors du cadre onusien ou encore d'initiatives ou programmes internationaux tels que l'initiative du rapporteur spécial des Nations Unies au droit à l'alimentation ou encore le Haut Comité à la Nutrition. S'ajoute évidemment à ce paysage, toute une série d'actions ou de programmes d'organisations agissant eux aussi à l'échelle globale (tels que ceux des organisations non-gouvernementales, des firmes multinationales, ou encore des programmes de coopération régionale). Cette grande diversité révèle un contexte d'absence de leadership affirmé d'une institution internationale en charge des questions de sécurité alimentaire, aucune d'entre elles n'ayant la légitimité suffisante pour couvrir la totalité du spectre. Mais elle révèle aussi une complexification croissante des questions globales avec des interdépendances accrues entre pays et des inter-relations de plus en plus fortes à gérer entres questions autrefois traitées selon un découpage sectoriel (Drezner, 2009; Biermann et al., 2008).

C'est dans ce contexte qu'il est intéressant de faire ressortir trois initiatives qui précisément cherchent à prendre en charge les enjeux de coordination sur les questions de sécurité 
alimentaire. Ces trois initiatives prétendent explicitement agir sur la qualité de la coordination internationale par un accroissement de la cohérence et de l'efficacité de cette coordination. Elles représentent trois formes différentes de dynamiques globales (pour une description plus détaillée, voir Lerin, Louafi 2009 et Margulis, 2001) :

- une de type intergouvernemental, centrée sur l'appareil des Nations Unies : la revitalisation du comité de la sécurité alimentaire (CSA) au sein de la $\mathrm{FAO}$;

- une autre conduite par les pays donateurs : la coordination au sein du G8/G20 autour d'un " Partenariat mondial pour I'agriculture, I'alimentation et la nutrition ";

- une autre enfin de type bureaucratique, mise en place par le Secrétaire des Nations Unies : la " High Level Task Force on Food security and nutrition " (HLTF) et son secrétariat, chargés de la coordination de l'ensemble des 20 agences des NU à l'échelle globale mais aussi sur le terrain dans certains pays cibles.

Nous faisons I'hypothèse ici que ces initiatives, par leur complémentarité, peuvent avoir une vertu intégratrice plutôt que celle de prolonger la fragmentation actuelle. En d'autres termes, elles peuvent être vues comme une étape vers la construction de la sécurité alimentaire comme une question globale.

Établi en 1974 dans le contexte de la crise alimentaire de 1974 suite au choc pétrolier, le CSA devait être un forum d'analyse et de suivi des politiques de sécurité alimentaire (Lele, 2009 ; Shaw, 2009). Il est toutefois resté cantonné à un rôle technique, centré sur les seuls aspects agricoles de disponibilité sans jamais réussir à affirmer un leadership ni sur le plan politique, ni analytique. Sa revitalisation porte précisément sur la nécessité d'établir, à défaut d'un leadership, une réel point de ralliement, sorte de plateforme où l'ensemble des acteurs concernés pourrait échanger, se coordonner voire élaborer des normes communes. Comme souligné dans le texte fondateur du "nouveau »CSA, I'objectif de cette revitalisation vise à en faire "(..) la principale plateforme internationale et intergouvernementale ouverte, regroupant un large éventail de parties prenantes ayant pris l'engagement de travailler ensemble de façon coordonnée et à l'appui de processus

Tableau 1. Prise en charge des socles institutionnels et discursifs par les trois méta-initiatives.

\begin{tabular}{|llll|}
\hline & CSA & G8/G20 & HLTF \\
\hline Agricole & +++ & ++ & + \\
\hline Commercial & $0 /+$ & + & + \\
\hline Humanitaire & $0 /+$ & ++ & + \\
\hline Droit & + & 0 & + \\
\hline Développement & - & +++ & + \\
\hline
\end{tabular}

CSA : Comité de la sécurité alimentaire

HTLF : High Level Task Force on Food security and nutrition

impulsés par les pays pour l'élimination de la faim et la garantie de la SA et nutritionnelle de l'ensemble de I'humanité ".

Mais en restant enchâssé au sein de la $\mathrm{FAO}$, le CSA prend le risque de rabattre systématiquement, consciemment ou non, la question de la sécurité alimentaire sur les questions agricoles. La présence de délégués dont le mandat et la légitimité portent sur les questions agricoles fait qu'ils ont moins d'aptitude à considérer les bénéfices à tirer d'une coordination avec d'autres problématiques ou $d$ 'autres secteurs: de manière schématique, on pourrait dire que l'environnement éloigne des préoccupations productivistes ou agro-exportatrices; que les préoccupations sanitaires engendrent des coûts de régulation supplémentaires dans la filière et menace la compétitivité, etc.

Par ailleurs, la FAO étant elle-même constituée de personnels ayant développé, de part la culture maison, leur expertise dans le domaine agricole, ils ont euxmêmes peu d'incitations (ou de légitimité) pour nouer des ponts avec les autres dimensions de la sécurité alimentaire. Certains le font néanmoins mais il s'agit davantage de démarches individuelles, souvent presque contre l'institution elle-même, plutôt que quelque chose de pensé stratégiquement et valorisé par l'institution.

La HLTF se démarque clairement ici en adoptant résolument une portée très large en termes de thématiques couvertes sous la bannière "sécurité alimentaire " (tableau 1). Par construction même, elle recouvre toutes les dimensions portées par l'ensemble des agences des Nations Unies spécialisées plus quel-

\footnotetext{
${ }^{1}$ Voir document CFS:2009/2 Rev 2, § 4, FAO, Rome.
}

ques autres organisations internationales. En pointant la nécessité d'impliquer l'ensemble des agences des Nations Unies pour résoudre le problème de sécurité alimentaire, la HLTF adresse un message fort sur le besoin de structures transversales pour répondre à des défis globaux au-delà des découpages sectoriels d'agences spécialisées des NU. Toutefois, il est clair que la présence des chefs d'agences dans cette task force ne garantit pas à elle seule la réalisation effective de cette prise en charge de I'inter-sectorialité. C'est au sein de l'équipe spéciale de coordination qu'elle s'exprime davantage avec le travail de coordination sur le terrain.

En ce qui concerne le G8/G20, la multidimensionnalité de la sécurité alimentaire n'a pas été initialement prise en charge, l'accent étant essentiellement mis sur la dimension financière à la fois pour de l'aide d'urgence et de l'investissement structurel. C'est donc avant tout une tentative de renforcer la coordination des interventions des donateurs en assurant la durabilité du soutien au-delà de l'aide ponctuelle à des fins humanitaires (Bachelier, 2011). Les présidences successives depuis la crise de 2008 n'ont eu de cesse de montrer qu'elles étaient attachées à réaliser les promesses financières. Mais la coordination ne s'est pas arrêtée là et la question de la volatilité des prix est apparue sur l'agenda, montrant un souci $d^{\prime}$ articulation entre les questions agricoles et commerciales dans un contexte d'enlisement du cycle de Doha. Mais I'apparition sur l'agenda ne signifie pas la prise en charge effective de la question et peu de nouvelles mesures ou solutions ont résulté de ces discussions.

En plus des différences de traitement sur le fond entre ces trois méta-initiatives, des différences marquées existent sur le plan procédural. Alors qu'à la fois la 
HLTF et le G8 (même élargi au G20) se constituent clairement comme des clubs fermés (d'agences des NU dans un cas, d'Etats dans l'autre), le CSA reste une enceinte intergouvernementale de type universel (sont membres de droit tous les États membres de la FAO). Mais au-delà de cette légitimité formelle, la revitalisation a aussi consisté à s'ouvrir à l'ensemble des porteurs d'enjeux (Louafi, 2012). Ainsi, retrouve-t-on au sein du comité consultatif (advisory group), un panel représentatif des acteurs qui comptent dans la sécurité alimentaire : des représentants d'organisations non gouvernementales, d'organisations professionnelles, du secteur privé et des fondations, des organisations internationales "sœurs " et "amies " et des organisations financières internationales. Cette présence au sein du comité consultatif du CSA garantit une représentation de ces membres au-delà des réunions annuelles du CSA lui-même : ils interviennent dans le travail intersessionnel pour aider à fixer les orientations de travail et thématiques abordées.

Mais la réelle innovation du CSA reste sans nul doute la constitution du panel d'expert de haut niveau sur la sécurité alimentaire et la nutrition (Louafi, 2012) Bâti sur le modèle du Groupe d'experts intergouvernemental sur l'évolution du climat - Giec, ce panel est censé fournir des données et recommandations les plus objectives possibles dans un domaine marqué davantage par une prolifération d'expertises concurrentes (se référant à des socles discursifs différents) que par un déficit d'expertise. La constitution de ce panel doit permettre de faire émerger des débats et des solutions habituellement "filtrées " par les intérêts - réels ou supposés - des institutions en place ou des États. À ce titre, elle doit aussi permettre de confronter les différentes formes de savoirs et cultures politiques ("policy cultures") existantes, qu'elles proviennent du monde de la bureaucratie des organisations internationales, de la société civile, du monde économique ou du milieu académique. Si ce panel fonctionne comme prévu, il est destiné à devenir un élément clé pour jeter les bases d'une transversalité sur la sécurité alimentaire et trancherait réellement avec le mode de mobilisation de l'expertise au sein des deux autres métainitiatives. Au sein du G8/G20, il s'agit essentiellement d'une conception ins- trumentale de la connaissance, celle-ci étant rarement confrontée car directement mise, par les administrations nationales en charge, au service de leurs gouvernements en fonction d'un agenda prédéfini. Pour la HLTF, les choses sont plus nuancées. En elle-même, la task force ne permet ni la mobilisation de connaissances, ni l'élaboration de nouvelles idées. On reste dans de la coordination bureaucratique où les enjeux substantiels sont très souvent relégués au dernier plan. Par contre, la HLTF comprend également un processus plus ouvert pour la construction de son Plan Global d'Action (Comprehensive Framework of Action), à travers des consultations larges d'experts hors du cadre étatique ou onusien et à travers le travail de coordination mené par l'équipe spéciale qui travaille à cette coordination inter-agences sur le terrain. L'expérience accumulée localement est, potentiellement en tout cas, réinjectée dans le mécanisme d'apprentissage nécessaire à l'élaboration de ce même plan global $\mathrm{d}^{\prime}$ action. Mais ce potentiel d'innovation reste limité par une double contrainte : d'ordre bureaucratique tout d'abord avec la nécessité d'obtenir l'approbation de l'ensemble des agences; statutaire ensuite, cette coordination inter-agences ne pouvant exister au sein des Nations unies uniquement si elle limite son champ d'action aux aspects techniques et ne s'aventure pas dans le domaine des politiques publiques (policy), prérogative des États membres. Une telle frontière est évidemment hautement problématique pour un sujet foncièrement politique comme l'est la sécurité alimentaire.

\section{Conclusion}

Les méta-initiatives décrites sont donc des tentatives de coordination d'organisation qui sont elles-mêmes, dans leur majorité, des ensembles multilatéraux. II $s^{\prime}$ agit donc en quelque sorte de coordination de coordination, au niveau le plus élevé. On peut bien sûr être sceptique sur l'efficacité de ces intentions et de ces dispositifs et mettre en avant le fait que la malnutrition n'a pas été éradiquée (Banerjee et Duflo, 2011). II nous semble cependant qu'il faut les tenir pour ce qu'elles sont : une certaine volonté de construire de manière cohérente la question de la sécurité alimentaire mondiale et de lui donner, d'une façon ou d'une autre le statut de bien public global - au même titre que l'environnement. La question des socles discursifs et institutionnels n'est évidemment pas résolue par une telle intention. On peut estimer toutefois que ces coordinations donnent une chance à la constitution d'une communauté épistémique qui serait seule en mesure de "précipiter" (au sens chimique du terme) cette fragmentation en une question globale, unifié et traitée comme telle.

Même si la réussite de ce processus de coordination et de tentative de fabrication d'une action collective pour fournir un bien public global n'est pas donnée, ce n'est probablement pas en son sein qu'il faut chercher les arguments du scepticisme, c'est principalement dans le fait que le système économique international est aujourd'hui principalement, voire exclusivement piloté par les coordinations économiques, elles mêmes dérivées du colmatage, crise après crise, du système financier international.

\section{Conflits d'intérêts : aucun.}

\section{RÉFÉRENCES}

Abbot PC, Hurt C, Tyner WE. What's driving food prices. Oak Brook (II., USA): Farm Foundation. Issue report, 2008.

Alter KJ, Meunier S. The politics of international regime complexity. Perspectives on politics 2009; 7 : 13-24.

Arts B, Buizer M. Forests, discourses, institutions: A discursive-institutional analysis of global forest governance. Forest Policy and Economics 2009 ; 11 : 340-7.

Bachelier B. Le G20 agricole : une chance pour l'agriculture mondiale? Paris: FARM (Fondation pour l'Agriculture et la Ruralité dans le Monde), 2011. http://www.fondation-farm. org/IMG/pdf/e2011-05-24-g20agricole_light_ fr.pdf.

Banerjee A, Duflo E. More than 1 billion people are hungry in the world. The Food issue 2011 ; (may/june). http://www. foreignpolicy.com/articles/2011/04/25/more_ than_1_billion_people_are_hungry_in_the world.

Banque Mondiale. Rapport sur le développement dans le monde: I'agriculture au service du développement. Washington: Banque mondiale, 2008.

Bertini C, Glickman D (eds). 2011 Progress report on US leadership in global agricultural development. Chicago : The Chicago Council on global affairs. Global agricultural development initiative, 2011. 
Biermann F, Pattberg PH, van Asselt $\mathrm{H}$, Zelli F. Fragmentation of global governance architectures: The case of climate policy. Global governance working paper; n. 34, 2008. http://www.glogov.org/images/doc/WP34. pdf.

Boussard JM, Gérard F, Piketty MG. Libéraliser I'agriculture mondiale? Théories modèles et réalités. Montpellier: CIRAD, 2005.

Braun JV. The world food situation. New driving forces and required actions. Washington: IFPRI. Food policy report, 2007.

Braun JV. Food and financial crisis. Implications for agriculture and the poor. Washington: IFPRI. Food policy report, 2008.

Bricas N, Daviron B. De la hausse des prix au retour du "productionnisme " agricole: les enjeux du sommet sur la sécurité alimentaire de juin 2008 à Rome. Hérodote 2008 ; (131) : 31-9.

Brunel S, Lemaître F, Madaule S. La crise alimentaire qui vient. Economie politique $\left(L^{\prime}\right)$ 2009 ; (43) : 7-34. http://www.cairn.info/ revue-I-economie-politique-2009-3.htm.

Davis CL. Overlapping institutions in trade policy. Perspectives on politics 2009 ; $7: 25-31$.

de Janvry A, Sadoulet E. The global food crisis: Identification of the vulnerable and policy responses. Agricultural and research economics update $2008 ; 12$ : 18-21. Special issue : causes and consequences of the food price crisis.

De Schutter O. Rapport du Rapporteur spécial sur le droit à l'alimentation. Nations Unies. Assemblée générale. Conseil des droits de l'homme. $16^{\mathrm{e}}$ session. Point 3 de l'ordre $\mathrm{du}$ jour. Promotion et protection de tous les droits de I'homme, civils, politiques, économiques, sociaux et culturels, y compris le droit au développement, 2010.

Drèze J, Sen A. Hunger and public action. Oxford: Clarendon press. Wider studies in development economics, 1989.

Drezner DW. The power and peril of international regime complexity. Perspectives on politics $2009 ; 7$ : 65-70.

European Commission. Dir. Gen. for Agr. and Rur. Dev. High prices on agricultural commodity market: situation and prospects. A review of causes of high prices and outlook for world agricultural markets. Bruxelles : European Commission, 2008.

Evans A. The feeding of the nine billion: global food security for the 21 st century: a Chatham House report. Londres: Chatham House, 2009.

FAO, OECD (eds). Price Volatility in Food and Agricultural Markets: Policy Responses. Paris : OCDE, 2011. http://www.oecd.org/ dataoecd/40/34/48152638.pdf.
Gérard F, Dorin B, Bélière JF, Diarra A, Keita $\mathrm{M}$, Dury S. Flambée des prix alimentaires internationaux : opportunite ou désastre pour les populations les plus pauvres? Montpellier : UMR MOISA. Working Paper ; n. 8, 2008.

Griffon M. Les multiples dimensions du problème alimentaire mondial. Etudes revue de culture contemporaine 2010 ; 413 : 595606.

Hafner-Burton EM. The power politics of regime complexity: Human rights trade conditionality in Europe. Perspectives on politics $2009 ; 7$ : 33-7.

Hoddinott J, Cohen MJ, Barrett CB. Renegotiating the Food Aid Convention: background, context, and issues. Global governance 2008 ; 14 : 283-304.

Hook D. Discourse, knowledge, materiality, history: Foucault and discourse analysis. Theory \& Psychology $2001 ; 11: 521$.

Hrabanski M. Souveraineté alimentaire : mobilisations collectives agricoles et instrumentalisations multiples d'un concept transnational. Revue Tiers-monde 2011 ; (207): 151-68.

IATP (Institute for Agriculture and Trade Policy). Commodities market speculation: the risk to food security and agriculture. Minneapolis (Minn, USA) :IATP, 2008.

IFPRI. Reaching Sustainable Food Security for All by 2020: Getting the priorities and responsibilities right. Washington: IFPRI, 2002.

Jacquet P, Pachauri RK, Tubiana L (Eds.). Regards sur la terre 2011. Paris: Armand Colin. Dossier: Océans : la nouvelle frontière, 2011.

Keohane RO, Victor DG. The regime complex for climate change. Perspectives on politics $2011 ; 9: 7-23$.

Lele U. Global food and agricultural institutions: The cosmology of international development assistance. Development policy review $2009 ; 27$ : 771-84.

Lerin F, Lemeilleur S, Petit M. Volatilité des prix internationaux agricoles et alimentaires et libéralisation en Afrique du Nord. 64. Paris: CIHEAM : 9-49. Options Méditerranéennes : Série B. Etudes et Recherches. 2009. http:// ressources.ciheam.org/om/pdf/b64/00801108. pdf.

Lerin F, Louafi S. "Tout bouge, rien ne change ?" De la difficulté à considérer la sécurité alimentaire comme un bien public global. Courrier de la planète $2009 ;(91)$ : 4-7. http://www.courrierdelaplanete.org/91/article1.php.

Lofgren H, Richards A. Food security, poverty, and economic policy in the Middle East and North Africa. In : Lofgren $\mathrm{H}$ (ed.), Food, agriculture, and economic policy in the Middle
East and North Africa. Emerald group publishing limited: 5-31. Research in Middle East economics ; n. 5, 2003.

Louafi S. Reform of the committee on food security: an opportunity for global governance? In : Jacquet Pierre, Pachauri Rajendra K, Tubiana Laurence (Eds.). Towards agricultural change? New Delhi : TERI, 2012 : 25860.

Margulis ME. The global food security crisis and multilateral cooperation: can international trade rules help? Paper presented to the GARNET annual conference, november 11 13, 2009.

Margulis ME. The evolving global governance of food security. Ottawa : Foreign Affairs and International Trade Canada. DFAIT policy staff, 2011. http://ssrn.com/abstract $=1823453$.

Maxwell D, Webb P, Coates J, Wirth J. Fit for purpose? Rethinking food security responses in protracted humanitarian crises. Food policy 2010 ; $35: 91-7$.

Maxwell S. Food security: a post-modern perspective. Food Policy 1996 ; 21 : 155-70.

McCalla AF. FAO in the Changing Global Landscape. Davis : University of California. Department of Agricultural and Resource Economics. Working paper ; n. 07-2006, 2007.

Mclntyre BD, Herren HR, Wakhungu J, Watson RT (Eds). Agriculture at a crossroads: International assessment of agricultural knowledge, science and technology for development (IAASTD): synthesis report with executive summary: a synthesis of the global and sub-global IAASTD reports. Washington: Island Press, 2009. http:// www.agassessment.org/reports/IAASTD/EN/ Agriculture $\% 20$ at $\% 20$ a $\% 20$ Crossroads Synthesis\%20Report\%20\%28English\%29.pdf.

Mitchell D. A note on rising food prices. Washington: World Bank. Policy research working paper ; n. 4682, 2008.

Oxfam. Au-delà des clivages: réformer la gouvernance mondiale sur la sécurité alimentaire. Paris: Oxfam. Note d'information, 2009.

Phillips N, Lawrence TB, Hardy C. Discourse and institutions. The Academy of Management Review 2004 ; 29 : 635-52.

Piesse J, Thirtle $C$. Three bubbles and a panic: An explanatory review of recent food commodity price events. Food Policy 2009 ; 34 : 119-29.

Pretty J, Sutherland W], Ashby J, et al. The top 100 questions of importance to the future of global agriculture. International journal of agricultural sustainability $2010 ; 8$ : 219-36.

Raustiala K, Victor DG. The regime complex for plant genetic resources. International organization $2004 ; 58: 277-309$. 
Risse-Kappen T, Ropp SC, Sikkink K (Eds). The power of human rights: International norms and domestic change. Cambridge: Cambridge University Press, 1999.

Robles $\mathrm{M}$, Torero $\mathrm{M}$, Von Braun J. When speculation matters. IFPRI Issue Brief 2009 ; $57: 1-7$.

Schmidt VA. Discursive institutionalism: The explanatory power of ideas and discourse. Annual review of political science 2008 ; 11 : 303-26.

Sen A. Poverty and famines: an essay on entitlement and deprivation. Oxford : Oxford university press, 2010.
Shapouri S, Peters M, Allen S, Rosen S, Baquedano F. Food security assessment, 201020. Washington : USDA. Economic Research Service, 2010.

Shaw DJ. World food security: a history since 1945. New York: Palgrave Macmillan, 2007.

Shaw DJ. Global food and agricultural institutions. Londres: Routledge. Routledge global institutions, 2009.

Summer A. The new bottom billion. The broker 2010 ; (23) : 4-8.

Testard-Vailland P. Alimentation mondiale. Les racines de la crise. Le journal du CNRS 2008 ; (224).
Timmer CP. Reflections on food crises past. Food policy $2010 ; 35: 1-11$.

Trostle R. Global agricultural supply and demand: Factors contributing to the recent Increase in food commodity prices. Washington : USDA-ERS. Outlook peport WRS0801, 2008.

Voituriez T. Hausse des prix de l'énergie, hausse des prix agricoles : quelles relations et implications à moyen-terme. IDDRI-IFRI, Working paper, 2009.

World B. Global economic prospects: Commodities at the crossroads. Washington : World Bank, 2009. 